\title{
Higher Nursing Care Level is Associated with Higher Incidence of Blood Culture Contamination in the Emergency Department: A Case-Control Study
}

\author{
Ayami Shigeno ( $\nabla$ shige.aya@gmail.com ) \\ Tokyo Bay Urayasu Ichikawa Medical Center \\ Yosuke Homma \\ Tokyo Bay Urayasu Ichikawa Medical Center \\ Taiga Matsumoto \\ Tokyo Bay Urayasu Ichikawa Medical Center \\ Shun Tanaka \\ Tokyo Bay Urayasu Ichikawa Medical Center \\ Ryuta Onodera \\ Tokyo Bay Urayasu Ichikawa Medical Center \\ Rentaro Oda \\ Tokyo Bay Urayasu Ichikawa Medical Center \\ Hiraku Funakoshi \\ Tokyo Bay Urayasu Ichikawa Medical Center
}

\section{Research Article}

Keywords: blood culture contamination, emergency department operations, geriatrics, infectious diseases

Posted Date: September 27th, 2021

DOl: https://doi.org/10.21203/rs.3.rs-875976/v1

License: (a) (i) This work is licensed under a Creative Commons Attribution 4.0 International License. Read Full License 


\section{Abstract \\ Background}

Blood culture is critical in treating infectious diseases, but contamination occurs in $0.6-12.5 \%$ of all samples. This leads to unnecessary intervention, inappropriate antibiotic use, and excess cost. Few studies have tackled patient factors, such as nursing care level, that could possibly affect contamination rates. Thus, this study aimed to explore the association between patients' nursing care levels and blood culture contamination.

\section{Methods}

This is a single centered, retrospective, case-control study of adult patients whose blood culture specimens were taken in the emergency department between April 2018 and July 2019. The study was conducted in a 344-bed, urban, acute care community hospital in Chiba prefecture, Japan. We included patients aged 20 years and above, with two or more sets of blood cultures. The case group included patients with false positive blood culture results with contamination; the control group included patients with true positive or true negative blood culture results without contamination. We randomly selected two control patients per case. Patients' age, sex, nursing care level, ambulance usage, housing status, Glasgow Coma Scale, and hospital arrival time were obtained from the patients' medical charts.

\section{Results}

Of the 5,130 patients, 686 patients got positive blood culture results. Of the 686 patients, 35 patients were included in the case group, and 70 were randomly selected from the non-contaminated group and included in the control. In multivariate analysis, patients with contaminated blood cultures had a higher nursing care level (odds ratio: $5.24 ; 95 \%$ confidence interval: 1.47 to $18.70 ; P=0.02$ ).

\section{Conclusions}

A higher nursing care level is associated with a higher incidence of blood culture contamination in the emergency department. Careful and appropriate procedures are required for patients with a higher nursing care level.

\section{Background}

Blood culture is a standard clinical procedure used to identify the source of infection and establish the appropriate treatment plan [1]. It is essential for detecting bacteremia, which is a critical health problem. For accurate identification of bacteremia, several guidelines recommend collecting two or more sets of samples for blood culture. [2,3] However, blood culture contamination occurs in $0.6-12.5 \%$ of samples [4], 
which leads to unnecessary intervention, inappropriate antibiotic use, and excess cost. The American Society of Microbiology recommends keeping the contamination rate under 3\% [2].

Previous studies showed that blood culture procedures, such as skin sterilization or puncture site selection, are associated with contamination [5-7]. Specimen collection site, inadequate skin preparation, and lack of sterile gloving contribute to contamination [8]. However, studies on patient factors are limited. We suspect that each patient's hygiene is varied, and it could affect blood culture contamination. The purpose of this research is to identify patient factors that affect blood culture contamination in the Emergency Department (ED).

In this study, we hypothesize that a higher nursing care level is associated with a higher incidence of blood culture contamination. Focusing on patients needing high levels of care and identifying factors associated with blood culture contamination may increase the accuracy and efficiency of blood cultures, especially among the older patients.

\section{Methods}

\subsection{Study design and setting}

This is a retrospective and case-control study conducted at the Tokyo Bay Urayasu Ichikawa Medical Center, a 344-bed, urban, acute care, community hospital in Japan. The ED of this hospital accepts approximately 10,000 ambulance arrivals, with an average of 25,000 patients per year [9]. The study was approved by the ethics committee of the Tokyo Bay Urayasu Ichikawa Medical Centre (approval number 429). Patient information was anonymized and de-identified prior to analysis, thus the informed consent of the patient was waived. This study also follows the STROBE statement on reporting case-control studies [10].

\subsection{Participants}

We included patients who had their blood culture specimens taken at the ED between April 2018 and July 2019. We excluded patients aged below 19 years and patients with only one set of blood cultures. The case group included patients who received false positive blood culture results due to contamination. The control group included patients with true positive or true negative blood culture results without contamination. We randomly selected two controls per case.

\subsection{Data collection}

Patients' age, sex, nursing care level, ambulance usage, housing status (home/hospital/care facility), GCS, and hospital arrival time (daytime was $8: 30$ am to $6 \mathrm{pm}$ and night-time was $6 \mathrm{pm}$ to $8: 30 \mathrm{am}$ ) were obtained from the patients' hospital electronic medical charts. Ambulance usage, housing status, and GCS were included because they are associated with the patients' severity of illness and ability to follow instructions during medical procedures $[11,12]$. The hospital arrival time was also included because it reflects crowding in the ED, which contributes to difficulties experienced during procedures [13]. As for housing status, people who have difficulties living at home by themselves or with their families tend to 
transfer to long-term care facilities. We inferred that the patients' housing status would reflect their activities of daily living (ADL) and cognitive function. The mentioned variables are categorical.

\subsection{Exposures and outcomes}

The primary exposure was nursing care level. We adopted the nursing care level classification from the Japanese Ministry of Health, Labor, and Welfare [14]. This classification system is for people aged over 65 years; the levels are based on their general condition, including ADL and cognitive function. Candidates are divided into seven levels [15]. Level 1 is the most independent and level 7 is the most dependent. In this study, we classified patients belonging to nursing care levels 6 and 7 as 'Higher Nursing Care Level' since most of them needed more care for their daily lives (Fig. 1).

The primary outcome of this study was blood culture contamination rate. We defined contamination as the presence of normal skin flora in a blood culture obtained from two or more sites [3]. The department of infection control, with board-certified infectious disease physicians, correlated independently the blood culture results with the patients' actual clinical course to determine the presence of an infection and the accuracy of the blood culture [16].

\subsection{Statistical analysis}

We evaluated the patients' characteristics and outcomes using the Fisher's Exact Test for binary variables, and the Mann-Whitney U Test for continuous variables. To determine the association between nursing care level and blood culture contamination, we constructed a multivariate logistic regression model, adjusting for higher nursing care levels, ambulance usage, housing status, and consciousness. To examine the robustness of our inference, we performed a sensitivity analysis comparing other nursing levels. This was done because the Japanese nursing care system has a service gap between levels 2 and 3 . People belonging to levels 1 and 2 are treated as if they can perform normal daily activities even if they need partial care or service for support. All P-values were two-tailed, with $\mathrm{P}<0.05$ considered statistically significant. The statistical analysis was performed with EZR (Saitama Medical Center, Jichi Medical University, Saitama, Japan), which is a graphical user interface for R 3.5.1 (R Foundation for Statistical Computing, Vienna, Austria) that enables statistical functions frequently used in biostatistics [17].

\subsection{Patient and Public Involvement}

Patients or the public were not involved in the design, conduct, reporting, or dissemination plans of our research.

\section{Results}

Between April 2018 and March 2019, 5,165 patients had specimens collected for blood culture in the ED. We excluded 35 patients according to the exclusion criteria described previously. Overall, 686 patients had positive blood culture results and 4,479 patients had negative blood culture results. Of the 686 blood culture positive patients, 35 had false positive results, and the contaminated blood cultures were included in the case group. Among patients with true positive or true negative results without contamination, 70 
were randomly selected and included in the control group (Figure 2). There were no missing data among patients who were included in the analysis.

Overall, the median patient age was 74 years (Interquartile Range: 62 to 85 ) and $75 \%$ were brought to the ED by ambulance. The baseline demographics and characteristics of patients are shown in Table 1.

Table 1. Explanatory variables for patients with contaminated and non-contaminated blood cultures.

\begin{tabular}{llll} 
& Contaminated, $\mathrm{n}(\%)$ & Not contaminated, $\mathrm{n}(\%)$ & P-value \\
\hline Aged over 65 years & $33(84.6)$ & $56(71.8)$ & 0.17 \\
\hline Sex (male) & $20(51.3)$ & $43(55.1)$ & 0.7 \\
\hline Higher nursing care level (level 6, 7) & $12(30.8)$ & $7(9.0)$ & 0.01 \\
\hline Ambulance usage & $34(87.2)$ & $50(64.1)$ & 0.01 \\
\hline Housing status (from home) & $27(69.2)$ & $65(83.3)$ & 0.09 \\
\hline GCS (below 13) & $14(35.9)$ & $18(23.1)$ & 0.19 \\
\hline M6 in GCS (could follow orders) & $27(69.2)$ & $68(87.2)$ & 0.02 \\
\hline Arrived at night-time* & $21(53.8)$ & $29(37.2)$ & 0.11
\end{tabular}

Abbreviations: GCS, Glasgow Coma Scale; M, Best motor response

*Defined as $6 \mathrm{pm}$ to $8: 30 \mathrm{am}$

On univariate analysis, patients whose blood cultures were suspected to be contaminated had a higher nursing care level ( $30.8 \%$ vs $9.0 \%, P=0.01)$, were brought to the ED by ambulance ( $87.2 \%$ vs $64.1 \%, P=0.01)$, and were unable to follow orders during procedures $(69.2 \%$ vs $87.2 \%, P=0.02)$.

On multivariate analysis, patients with higher nursing care levels (odds ratio [OR]: 5.24; $95 \%$ confidence interval [Cl]: 1.47 to 18.70; $P=0.02$ ) had a higher incidence of blood culture contamination (Table 2). On sensitivity analysis, patients with a nursing care level of 3 and above had a significantly higher incidence of blood culture contamination $(P=0.01)$.

Table 2. Multiple logistic regression analysis of the association between nursing care level and blood culture contamination.

\begin{tabular}{llll} 
& Odds ratio & $95 \% \mathrm{Cl}$ & P-value \\
\hline Unadjusted & 1.24 & $1.04-1.49$ & 0.02 \\
\hline Adjusted* & 5.24 & $1.47-18.70$ & 0.02
\end{tabular}

Abbreviation: $\mathrm{Cl}$, Confidence Interval 
*Adjusted for nursing care level, housing status, consciousness, and ambulance usage.

\section{Discussion}

\subsection{Principal findings}

Our study demonstrated that patients with a higher nursing care level had a higher incidence of blood culture contamination in the ED.

\subsection{Strengths and weakness of the study}

This study has several limitations. First, the study has a low generalizability because it is a single-center study. Despite this, our hospital accepts a wide range of patients regardless of illness severity or location, and the hospital's ambulance acceptance is $95 \%$ [9]. Additionally, the population pyramid and mean age in Chiba prefecture is similar to Japan's national population pyramid [25]. Second, people who were not using nursing care services were excluded, such as those living on the street or aged below 65 years. Third, our study did not consider the skin puncture site because of missing data and limitations in our medical chart ordering system. This is relevant because previous studies showed that the skin puncture site, particularly the femoral artery, affected contamination rates [7]. This is pertinent to elderly and critically ill patients who tend to have their specimens collected from the femoral artery because of difficulties in venipuncture. Further research is needed to evaluate the association between procedure factors such as puncture site and patients' factors including nursing care level to contamination rates.

\subsection{Strengths and weaknesses in relation to other studies}

Previous studies showed that improvements in blood culture specimen collection and standardized use of sterile gloves and/or chlorhexidine skin disinfectants reduces blood culture contamination $[2,6,7]$. However, few studies have explored the patient factors that may affect blood culture contamination rates. Our study focused on patients' physical and cognitive function and found that patients with lower ADL or cognitive function tended to have a higher risk for blood culture contamination.

Previous studies have shown that it is more difficult to conduct procedures in the ED compared to the inpatient wards and intensive care unit (ICU) [18]. Multiple studies on the incidence of central lineassociated bloodstream infections (CLABSI) showed that CLABSI rates in the ED were similar to those reported in the ICU $[19,20]$. These studies involved younger patients (median age $=60$ years), whereas our study involved older patients and may be more relevant to the older population.

\subsection{Meaning of the study}


Our study adopted the Japanese nursing care level classification that uses both ADL and cognitive function to assess a person's ability to maintain their hygiene. Some previous research showed there are associations between oral hygiene and elderly people's ADL or cognitive functions [24]. We hypothesized that people with a lower ADL or cognitive function had difficulties maintaining their hygiene, and it may affect blood contamination rate in the ED.

Since most patients at the ED come from their own homes, the patients' hygiene before their hospital visit may affect the blood culture contamination rate. Several studies on dental health showed that oral hygiene was associated with ADL scores among the homebound older patients. The studies implied that older patients with lower $A D L$ scores needed more support for daily personal care than those with higher ADL scores [21]. Additionally, other studies showed that $10 \%$ of the older patients with dementia had resistanceto-care behavior, including physical and/or psychological resistance. People with dementia may show abhorrence and aggressive behavior in response to the invasion of their personal space $[22,23]$. This adds to the difficulty of maintaining hygiene.

Guidelines from multiple organizations recommend a systems-based approach comprising education, procedure checklists, hand hygiene, use of sterile gloving, avoidance of femoral catheter insertion, and use of chlorhexidine-alcohol skin disinfectants $[2,6,7]$. Since the older patients need more medical care, they may undergo more frequent medical procedures, such as blood extractions for culture. These patients tend to have difficulties following orders, and more resources like manpower are needed to complete each procedure successfully [21]. Since early administration of appropriate empirical antibiotic therapy decreases mortality from bacteremia [4], a more careful, appropriate blood culture procedure is required.

\section{Conclusions}

Higher nursing care level is associated with higher incidences of blood culture contamination in ED. Although further researches are warranted, a more careful, appropriate procedure is required.

\section{List Of Abbreviations}

Cl: confidence interval

ED: emergency department

OR: odds ratio

\section{Declarations}

\section{Ethics approval and consent to participate:}

The study was approved by the ethics committee of the Tokyo Bay Urayasu Ichikawa Medical Centre (approved number 429). Patient information was anonymized and de-identified prior to analysis, thus the informed consent of the patient was waived. 
Consent for publication:

The same as above.

\section{Availability of data and materials:}

Extra data is available by emailing shige.aya@gmail.com

\section{Competing interests:}

The authors declare no competing interests.

\section{Funding:}

The study received no funding.

\section{Authors' contributions:}

AS, YH, TM, ST, RO and HF conceived and designed the study. AS, YH and HF supervised the conduct of the study and data collection. $\mathrm{YH}$ and $\mathrm{RO}$ undertook managed the data, including quality control. AS, $\mathrm{YH}$ and $\mathrm{HF}$ provided statistical advice on study design and analyzed the data. AS, YH and HF drafted the manuscript, and all authors contributed substantially to its revision. HF takes responsibility for the paper as a whole.

\section{Acknowledgements:}

None.

\section{References}

1. Towns ML, Jarvis WR, Hsueh PR. Guidelines on blood cultures. J Microbiol Immunol Infect. 2010;43:347-9. doi:10.1016/S1684-1182(10)60054-0.

2. Clinical practice guideline: prevention of blood culture contamination. J Emerg Nurs 2018;44:285.e1285.e24. doi:https://doi.org/10.1016/j.jen.2018.03.019.

3. Dawson S. Blood culture contaminants. J Hosp Infect. 2014;87:1-10. doi:10.1016/j.jhin.2014.02.009.

4. Lee CC, Lee CH, Chuang MC, Hong MY, Hsu HC, Ko WC. Impact of inappropriate empirical antibiotic therapy on outcome of bacteremic adults visiting the ED. Am J Emerg Med. 2012;30:1447-56. doi:10.1016/j.ajem.2011.11.010.

5. Story-Roller E, Weinstein MP. Chlorhexidine versus tincture of iodine for reduction of blood culture contamination rates: a prospective randomized crossover study. J Clin Microbiol. 2016;54:3007-9. doi:10.1128/JCM.01457-16.

6. Snyder SR, Favoretto AM, Baetz RA, Derzon JH, Madison BM, Mass D, et al. Effectiveness of practices to reduce blood culture contamination: a laboratory medicine best practices systematic review and 
meta-analysis. Clin Biochem. 2012;45:999-1011. doi:10.1016/j.clinbiochem.2012.06.007.

7. Liang SY, Riethman M, Fox J. Infection prevention for the emergency department: out of reach or standard of care? Emerg Med Clin North Am. 2018;36:873-87. doi:10.1016/j.emc.2018.06.013.

8. Self WH, Mickanin J, Grijalva CG, Grant FH, Henderson MC, Corley G, et al. Reducing blood culture contamination in community hospital emergency departments: a multicenter evaluation of a quality improvement intervention. Acad Emerg Med. 2014;21:274-82. doi:10.1111/acem.12337.

9. Tokyo Bay Urayasu Ichikawa Medical Center. https://tokyobay-mc.jp/. Accessed 10 Oct 2020.

10. von Elm E, Altman DG, Egger M, Pocock SJ, Gøtzsche PC, Vandenbroucke JP, et al. The strengthening the reporting of observational studies in epidemiology (STROBE) statement: guidelines for reporting observational studies. Int J Surg. 2014;12:1495-9. doi:10.1016/j.ijsu.2014.07.013.

11. Medical Advisory Secretariat. Caregiver- and patient-directed interventions for dementia: an evidencebased analysis. Ont Health Technol Assess Ser. 2008;8:1-98.

12. Laver K, Dyer S, Whitehead C, Clemson L, Crotty M. Interventions to delay functional decline in people with dementia: a systematic review of systematic reviews. BMJ Open. 2016;6. doi:10.1136/bmjopen2015-010767.

13. Carter EJ, Pouch SM, Larson EL. The relationship between emergency department crowding and patient outcomes: a systematic review. J Nurs Scholarsh. 2014;46:106-15. doi:10.1111/jnu.12055.

14. Overview of the long-term nursing care insurance system. Ministry of Health, Labor and Welfare. https://www.mhlw.go.jp/stf/seisakunitsuite/bunya/hukushi_kaigo/kaigo_koureisha/gaiyo/index.html. Accessed 10 Oct 2020.

15. Long-term care insurance in Japan. https://www.mhlw.go.jp/english/topics/elderly/care/2.html. Accessed 10 Oct 2020.

16. Doern GV, Carroll KC, Diekema DJ, Garey KW, Rupp ME, Weinstein MP, et al. A comprehensive update on the problem of blood culture contamination and a discussion of methods for addressing the problem. Clin Microbiol Rev. 2020;33. doi:10.1128/CMR.00009-19.

17. Kanda Y. Investigation of the freely available easy-to-use software 'EZR' for medical statistics. Bone Marrow Transplant. 2013;48:452-8. doi:10.1038/bmt.2012.244.

18. Lee CC, Lee NY, Chuang MC, Chen PL, Chang CM, Ko WC. The impact of overcrowding on the bacterial contamination of blood cultures in the ED. Am J Emerg Med. 2012;30:839-45. doi:10.1016/j.ajem.2011.05.026.

19. Theodoro D, Olsen MA, Warren DK, McMullen KM, Asaro P, Henderson A, et al. Emergency department central line-associated bloodstream infections (CLABSI) incidence in the era of prevention practices. Acad Emerg Med. 2015;22:1048-55. doi:10.1111/acem.12744.

20. Lemaster CH, Schuur JD, Pandya D, Pallin DJ, Silvia J, Yokoe D, et al. Infection and natural history of emergency departmentplaced central venous catheters. Ann Emerg Med. 2010;56. doi:10.1016/j.annemergmed.2010.05.033.

21. Ćatović A, Bergman V, Ćatić A. Qualitative evaluation of elderly home residents' fixed and removable prostheses in relation to the ADL index. J Dent. 2003;31:3-8. doi:10.1016/S0300-5712(02)00129-X. 
22. Konno R, Kang HS, Makimoto K. The best evidence for minimizing resistance-to-care during assisted personal care for older adults with dementia in nursing homes: a systematic review. JBI Database Syst Rev Implement Rep. 2012;10:4622-32. doi:10.11124/jbisrir-2012-431.

23. Patnode CD, Perdue LA, Rossom RC, Perdue LA, Burda BU, Thompson M, et al. Screening for cognitive impairment in older adults: an evidence update for the U.S. preventive services task force. Rockville (MD): Agency for Healthcare Research and Quality (US); 2020.

24. Morishita M, Takaesu Y, Miyatake K, Shinsho F, Fujioka M. Oral health care status of homebound elderly in Japan. J Oral Rehabil. 2001;28:717-20. doi:10.1046/j.1365-2842.2001.00713.x.

25. Overview of demographic statistics of Chiba prefecture 2018. 2020. https://www.pref.chiba.lg.jp/kenshidou/toukeidata/kakushukousei/jinkoudoutai/h30_gaikyou.html. Accessed 10 Oct 2020.

\section{Figures}

\section{Level General condition}

1 Need partial support for daily activity and care.

2 Need support for walking or standing.

3 Add to level 2, cognitive dysfunction or problematic behavior.

4 Need help for eating or excretion.

5 Cannot stand up and move by oneself.

6 Cannot excrete by oneself, severe dementia.

7 Cannot eat by oneself.

\section{Figure 1}

Nursing care level in Japan 


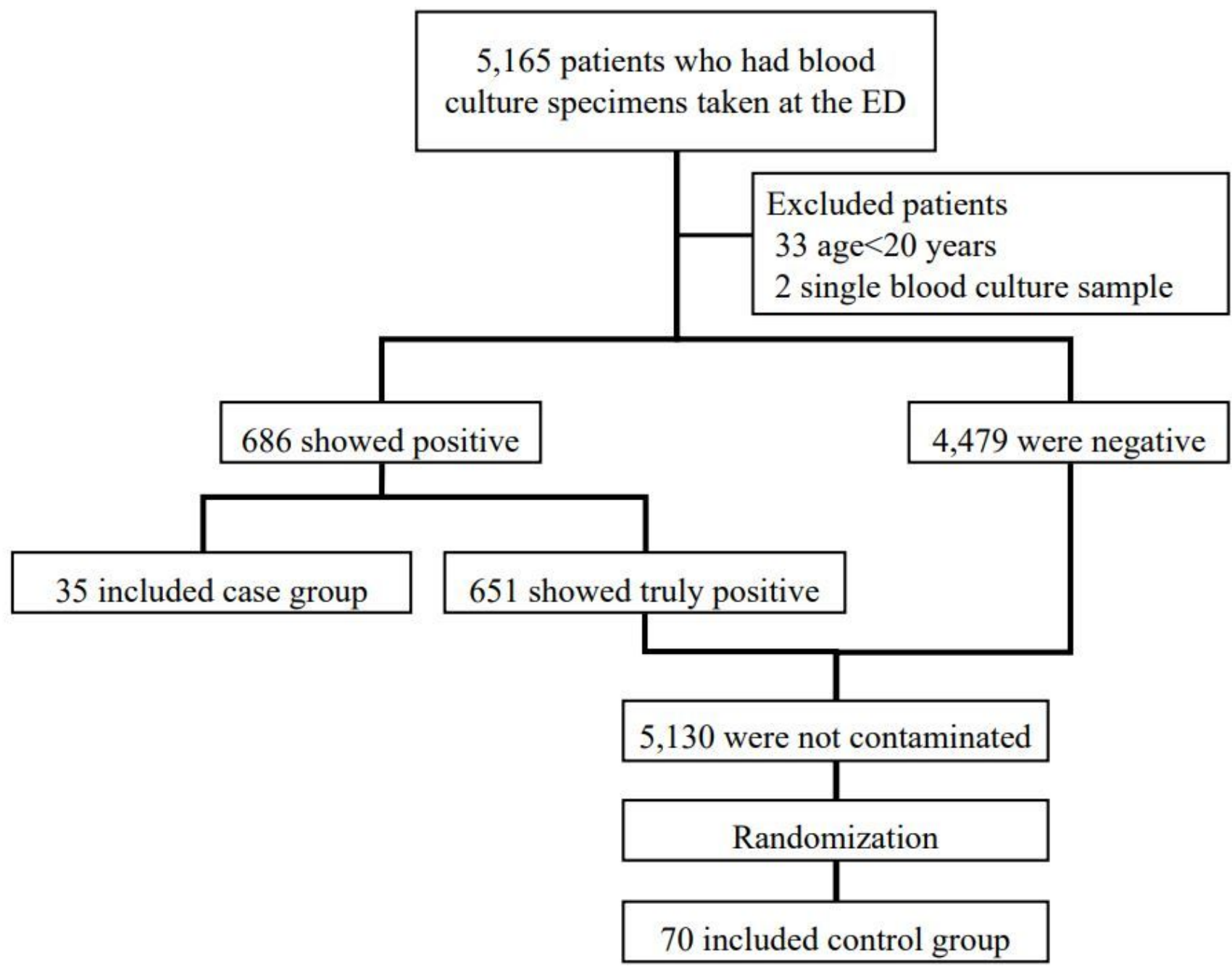

Figure 2

Selection of participants 